\title{
Effect of Site Specific Nutrient Management in Potato based Sequential Cropping Systems on Microbial Population Dynamics
}

\author{
M. H. Manjunatha ${ }^{1 *}$, S. Bhaskar ${ }^{2}$, B. C. Mallesha ${ }^{1}$, N. N. Lingaraju ${ }^{1}$ and B. Basavaraja ${ }^{1}$ \\ ${ }^{1}$ Department of Agronomy, UAS, GKVK, Bangalore, India \\ ${ }^{2}$ General (AAGCC), ICAR, New Delhi, India \\ *Corresponding author
}

\section{A B S T R A C T}

\section{Keywords}

Cropping system, Microbial

properties, Potato, SSNM

\section{Article Info}

Accepted:

10 January 2021

Available Online:

10 February 2021
A field experiments were conducted on the "Effect of site specific nutrient management in potato based sequential cropping systems on microbial population dynamics" in the premises of Agricultural Research Station (ARS), Madenur, Hassan Taluk and District during Kharif and Rabi 2017 and 2018. Experiment consisting of twelve treatments $\left(T_{1}\right.$ : $100 \% \mathrm{~K}$ through $\mathrm{KCl}+\mathrm{NP}$ through fertilizers for targeted yield of $20 \mathrm{tha}^{-1}, \mathrm{~T}_{2}: 100 \% \mathrm{~K}$ through $\mathrm{K}_{2} \mathrm{SO}_{4}+\mathrm{NP}$ through fertilizers for targeted yield of $20 \mathrm{tha}^{-1} \mathrm{~T}_{3}: 100 \% \mathrm{~K}$ through Bio-K + balance NP through fertilizers for targeted yield of $20 \mathrm{t} \mathrm{ha}^{-1,} \mathrm{~T}_{4}: 50 \% \mathrm{~K}$ through Bio-K $+50 \% \mathrm{~K}$ through $\mathrm{KCl}+$ balance NP through fertilizers for targeted yield of $20 \mathrm{t}$ ha ${ }^{1,} \mathrm{~T}_{5}: 50 \% \mathrm{~K}$ through Bio- $\mathrm{K}+50 \% \mathrm{~K}$ through $\mathrm{K}_{2} \mathrm{SO}_{4}+$ balance NP through fertilizers for targeted yield of $20 \mathrm{t} \mathrm{ha}^{-1}, \mathrm{~T}_{6}: 100 \% \mathrm{~K}$ through $\mathrm{KCl}+\mathrm{NP}$ through fertilizers for targeted yield of $25 \mathrm{t} \mathrm{ha}^{-1,} \mathrm{~T}_{7}: 100 \% \mathrm{~K}$ through $\mathrm{K}_{2} \mathrm{SO}_{4}+\mathrm{NP}$ through fertilizers for targeted yield of $25 \mathrm{tha}^{-1,} \mathrm{~T}_{8}: 100 \% \mathrm{~K}$ through Bio-K + balance NP through fertilizers for targeted yield of $25 \mathrm{t} \mathrm{ha}^{-1,} \mathrm{~T}_{9}: 50 \% \mathrm{~K}$ through Bio- $\mathrm{K}+50 \% \mathrm{~K}$ through $\mathrm{KCl}+$ balance NP through fertilizers for targeted yield of $25 \mathrm{t} \mathrm{ha}^{-1,} \mathrm{~T}_{10}: 50 \% \mathrm{~K}$ through Bio-K $+50 \% \mathrm{~K}$ through $\mathrm{K}_{2} \mathrm{SO}_{4}+$ balance NP through fertilizers for targeted yield of $25 \mathrm{t} \mathrm{ha}^{-1,} \mathrm{~T}_{11}$ : Recommended dose of fertilizers alone $\left(75: 75: 100 \mathrm{~kg}\right.$ NPK ha $\left.{ }^{-1}\right), \mathrm{T}_{12}$ : Package of Practice recommendation, UAS, B)and replicated thrice. Among the soil biological properties, microbial activity viz., bacteria, fungi and actinomycetes count after harvest of potato was significantly higher in treatments receiving $100 \% \mathrm{~K}$ through Bio-K + balance NP through fertilizers for targeted yield of $25 \mathrm{t} \mathrm{ha}^{-1}\left(10.27 \times 10^{6} \mathrm{Cfu} \mathrm{g}^{-1}, 6.25 \times 10^{4} \mathrm{Cfu} \mathrm{g}^{-1}\right.$ and $4.02 \mathrm{x}$ $10^{2} \mathrm{Cfu} \mathrm{g}^{-1}$, respectively) across all the treatments.

\section{Introduction}

Among several factors deciding the crop productivity over a specific time and area, nutrients supply plays a key role after water. The nutrient required by the crop either in larger or smaller quantities decides the productivity by its role in plant metabolic activities. The current and generalized fertilizer recommendations which were developed decades ago are losing their applicability due to the variability of soil fertility both in spatial and temporal dimensions. In addition to this, high crop 
removal coupled with less addition of nutrients by farmers has resulted in large scale depletion of macro and micro nutrients in the cultivated soil leading to emergence of deficiency in soil and crops, thus causing a shift in fertility from high to medium or medium to low nutrient status over the years. Further, the crop requirement for various nutrients vary greatly among fields, years and seasons as a result of variations in climate, crop growing conditions and also due to differences in crop and soil management practices. Thus, the current and generalized fertilizer recommendations which were developed decades ago were no longer valid and there is a need for finding a novel approach of nutrient management which takes into account of both field and crop specific requirements of nutrients.

Recent advances in nutrient management research have come out with an advanced technique of supplying nutrient to crop according to variability in soil over space and requirement during a particular growth period. The approach is termed as 'site specific nutrient management (SSNM).The novel approach of SSNM involves sciencebased principles for guiding the judicious and efficient application of fertilisers as and when needed by crops. It recognizes the inherent spatial variability associated with soil during crop production and provides guidelines for optimal use of indigenous nutrients originating from soil, plant residues, manures and irrigation water. Thus, SSNM considers indigenous nutrient supply of the soil and productivity and targets the capability of sustaining higher yields on one hand, and assured restoration of soil fertility on the other.

Cropping system is generally practiced on small farms with limited resources and it has been observed to enhance yields with greater stability in a variety of crop combinations.
Moreover, these systems is known by less use of inputs, namely, fertilizers, plant protection chemical and thus healthy, safe and high quality food under ecologically sound production system. On-farm biodiversity is also promoted by diversification of crops through crop rotation and intercropping systems resulting in variation of diet and more net return, higher level of production stability, proper utilization of limited resources human labour-force under low levels of technological intervention and all these ultimately lead to achieve production sustainability in agriculture. At present in Hassan district, potato-finger millet or potato-maize cropping sequence is gaining importance as dominant cropping systems. Since both potato and maize are exhaustive crops, maintaining both crop and soil productivity is one of the crucial factor for attaining higher yield.

Today, roots and tubers are the third largest carbohydrate food source in the world, with potatoes representing nearly half of all root crops consumed (Anon., 2018). Potatoes contribute key nutrients to the diet including vitamin $\mathrm{C}$, potassium, and dietary fibre (McGill et al., 2013). In fact, potatoes have a more favourable overall nutrient-to-price ratio than many other vegetables and are an important staple worldwide (Drewnowski, 2013). Even though the crop is known for its hardiness, its productivity under large scale production is decided by water and nutrient management techniques followed. Numerous studies document the crop response to $\mathrm{N}, \mathrm{P}$, and $\mathrm{K}$ fertilizer application. Yield and tuber quality are affected by both excess and lower application of nutrients. While, the negative impacts of over application of nutrients are attributed to salt damage (Bilski et al., 1988), disease (Somani and Shekhawat, 1988) and physiological changes (Westermann et al., 1994), lower availability of nutrients alter the crop productivity by influencing crop water relationships, variability of transpiration rates 
and increments in plant body temperature (Yli-Halla et al., 1987). Hence, keeping in view of the importance of nutrient management and its role in crop productivity, experiments were conducted to study the effect of site specific nutrient management in potato based sequential cropping systems on microbial population dynamics.

\section{Materials and Methods}

Field experiments were conducted on the "Effect of site specific nutrient management in potato based sequential cropping systems on microbial population dynamics" in the premises of Agricultural Research Station (ARS), Madenur, Hassan Taluk and District during Kharif and Rabi 2017 and 2018. The experimental plots of kharif season were divided into two plots to raise finger millet and field bean crops with recommended dose of fertilizers to study the effect of SSNM on succeeding crops. The treatment details consisting of twelve treatments $\left(\mathrm{T}_{1}: 100 \% \mathrm{~K}\right.$ through $\mathrm{KCl}+\mathrm{NP}$ through fertilizers for targeted yield of $20 \mathrm{t} \mathrm{ha}^{-1}, \mathrm{~T}_{2}: 100 \% \mathrm{~K}$ through $\mathrm{K}_{2} \mathrm{SO}_{4}+\mathrm{NP}$ through fertilizers for targeted yield of $20 \mathrm{t} \mathrm{ha}^{-1,} \mathrm{~T}_{3}: 100 \% \mathrm{~K}$ through Bio-K + balance NP through fertilizers for targeted yield of $20 \mathrm{tha}^{-1,} \mathrm{~T}_{4}: 50$ $\% \mathrm{~K}$ through Bio- $\mathrm{K}+50 \% \mathrm{~K}$ through $\mathrm{KCl}+$ balance NP through fertilizers for targeted yield of $20 \mathrm{t} \mathrm{ha}^{-1,} \mathrm{~T}_{5}: 50 \% \mathrm{~K}$ through Bio-K + $50 \% \mathrm{~K}$ through $\mathrm{K}_{2} \mathrm{SO}_{4}+$ balance NP through fertilizers for targeted yield of $20 \mathrm{t} \mathrm{ha}^{-1}$, $\mathrm{T}_{6}: 100 \% \mathrm{~K}$ through $\mathrm{KCl}+\mathrm{NP}$ through fertilizers for targeted yield of $25 \mathrm{t} \mathrm{ha}^{-1}$, $\mathrm{T}_{7}: 100 \% \mathrm{~K}$ through $\mathrm{K}_{2} \mathrm{SO}_{4}+\mathrm{NP}$ through fertilizers for targeted yield of $25 \mathrm{t} \mathrm{ha}^{-1,} \mathrm{~T}_{8}$ : $100 \% \mathrm{~K}$ through Bio-K + balance NP through fertilizers for targeted yield of $25 \mathrm{t}$ ha $^{-1,} \mathrm{~T}_{9}: 50 \% \mathrm{~K}$ through Bio-K $+50 \% \mathrm{~K}$ through $\mathrm{KCl}+$ balance NP through fertilizers for targeted yield of $25 \mathrm{t} \mathrm{ha}^{-1,} \mathrm{~T}_{10}: 50 \% \mathrm{~K}$ through Bio-K $+50 \% \mathrm{~K}$ through $\mathrm{K}_{2} \mathrm{SO}_{4}+$ balance NP through fertilizers for targeted yield of $25 \mathrm{tha}^{-1,} \mathrm{~T}_{11}$ : Recommended dose of fertilizers alone (75:75:100 kg NPK ha $\left.{ }^{-1}\right), \mathrm{T}_{12}$ : Package of Practice recommendation, UAS, B) with three replication. The experimental data obtained were subjected to statistical analysis adopting Fisher's method of Analysis of Variance (ANOVA) as outlined by Gomez and Gomez (1984). The level of significance used for $F$ test was 5 per cent. Critical difference (CD) values are given in the table at 5 per cent level of significance, wherever the " $F$ " test was significant at 5 per cent level. Pooled analysis of two years data was analysed using Additive Main Multiplicative Interaction (AMMI) method by Felipe de Mendibur (2020).

\section{Results and Discussion}

Significantly higher bacterial population was observed in soil after harvest of potato in plot which received $100 \% \mathrm{~K}$ through Bio-K + balance NP through fertilizers for targeted yield of $25 \mathrm{t} \mathrm{ha}^{-1}\left(10.27 \times 10^{6} \mathrm{Cfu} \mathrm{g}^{-1}\right)$ and it was on par with $50 \% \mathrm{~K}$ through Bio-K +50 $\% \mathrm{~K}$ through $\mathrm{K}_{2} \mathrm{SO}_{4}+$ balance $\mathrm{NP}$ through fertilizers for targeted yield of $25 \mathrm{t} \mathrm{ha}^{-1}$ (10.05 $\mathrm{x} 10^{6} \mathrm{Cfu} \mathrm{g}^{-1}$ ) as compared to other treatments.. Significantly lower bacterial population was observed in recommended dose of fertilizers alone $\left(2.65 \times 10^{6} \mathrm{Cfu} \mathrm{g}^{-1}\right)$ (Table 1). Fungal population was significantly higher with $100 \% \mathrm{~K}$ through Bio-K + balance NP through fertilizers for targeted yield of $25 \mathrm{t} \mathrm{ha}^{-1}\left(6.25 \times 10^{4} \mathrm{Cfu} \mathrm{g}^{-1}\right)$ and it was found to be on par with $50 \% \mathrm{~K}$ through Bio-K $+50 \% \mathrm{~K}$ through $\mathrm{K}_{2} \mathrm{SO}_{4}+$ balance NP through fertilizers for targeted yield of $25 \mathrm{t}$ $\mathrm{ha}^{-1}\left(6.06 \times 10^{4} \mathrm{Cfu} \mathrm{g}^{-1}\right), 100 \% \mathrm{~K}$ through $\mathrm{KCl}+\mathrm{NP}$ through fertilizers for targeted yield of $25 \mathrm{t} \mathrm{ha}^{-1}\left(5.92 \times 10^{4} \mathrm{Cfu} \mathrm{g}^{-1}\right)$ and $100 \% \mathrm{~K}$ through $\mathrm{K}_{2} \mathrm{SO}_{4}+\mathrm{NP}$ through fertilizers for targeted yield of $25 \mathrm{t} \mathrm{ha}^{-1}\left(5.75 \times 10^{4} \mathrm{Cfu} \mathrm{g}^{-1}\right)$ as compared to other treatments. Significantly lower fungal population was observed in recommended dose of fertilizers alone $(2.55 \mathrm{x}$ 
$10^{4} \mathrm{Cfu} \mathrm{g}^{-1}$ ) (Table 1). Significantly higher actinomycetes population was observed in soil after harvest of potato in plot which received $100 \% \mathrm{~K}$ through Bio-K + balance NP through fertilizers for targeted yield of 25 $\mathrm{t} \mathrm{ha}^{-1}\left(4.02 \times 10^{2} \mathrm{Cfu} \mathrm{g}^{-1}\right)$ and it was on par with $50 \% \mathrm{~K}$ through Bio-K $+50 \% \mathrm{~K}$ through $\mathrm{K}_{2} \mathrm{SO}_{4}+$ balance $\mathrm{NP}$ through fertilizers for targeted yield of $25 \mathrm{t} \mathrm{ha}^{-1}(3.90 \mathrm{x}$ $10^{2} \mathrm{Cfu} \mathrm{g}^{-1}$ ) as compared to other treatments. Significantly lower actinomycetes population was observed in recommended dose of fertilizers alone $\left(1.85 \times 10^{2} \mathrm{Cfu} \mathrm{g}^{-1}\right)$ (Table 1). Similar findings were also reported by Corne et al., (2017) and Cui et al., (2018).

Significantly higher bacterial population was observed in soil after harvest of finger millet with $100 \% \mathrm{~K}$ through Bio-K + balance NP through fertilizers for targeted yield of $25 \mathrm{t}$ $\mathrm{ha}^{-1}\left(12.40 \times 10^{5} \mathrm{Cfu} \mathrm{g}^{-1}\right)$ and it was on par with $50 \% \mathrm{~K}$ through Bio-K $+50 \% \mathrm{~K}$ through $\mathrm{K}_{2} \mathrm{SO}_{4}+$ balance NP through fertilizers for targeted yield of $25 \mathrm{tha}^{-1}(12.20$ $\mathrm{x} 10^{5} \mathrm{Cfu} \mathrm{g}^{-1}$ ) as compared to other treatments.. Significantly lower bacterial population was observed in recommended dose of fertilizers alone $\left(2.50 \times 10^{5} \mathrm{Cfu} \mathrm{g}^{-1}\right)$ (Table 2).

Table.1 Microbial population in soil after harvest of potato as influenced by site specific nutrient management in potato based sequential cropping system

\begin{tabular}{|c|c|c|c|c|c|c|c|c|c|}
\hline \multirow[t]{2}{*}{ Treatments } & \multicolumn{3}{|c|}{ Bacteria (x 10 ${ }^{6} \mathrm{Cfu} \mathrm{g}^{-1}$ ) } & \multicolumn{3}{|c|}{ Fungi $\left(\times 10^{4}\right.$ Cfu g $\left.^{-1}\right)$} & \multicolumn{3}{|c|}{ Actnomycetes (x 10 $\left.{ }^{2} \mathrm{Cfu} \mathrm{g}^{-1}\right)$} \\
\hline & 2017 & 2018 & Pooled & 2017 & 2018 & Pooled & 2017 & 2018 & Pooled \\
\hline $\mathbf{T}_{1}$ & 3.50 & 3.93 & 3.72 & 3.10 & 3.60 & 3.35 & 2.10 & 2.20 & 2.15 \\
\hline $\mathbf{T}_{2}$ & 3.30 & 3.50 & 3.40 & 3.00 & 3.52 & 3.26 & 2.00 & 2.12 & 2.06 \\
\hline $\mathbf{T}_{3}$ & 4.75 & 5.00 & 4.88 & 4.15 & 4.50 & 4.33 & 2.85 & 2.90 & 2.88 \\
\hline $\mathbf{T}_{4}$ & 3.90 & 4.73 & 4.32 & 5.00 & 5.30 & 5.15 & 2.10 & 2.60 & 2.35 \\
\hline $\mathbf{T}_{5}$ & 4.50 & 5.90 & 5.20 & 4.32 & 4.70 & 4.51 & 2.80 & 2.91 & 2.86 \\
\hline$T_{6}$ & 5.20 & 6.10 & 5.65 & 5.80 & 6.03 & 5.92 & 2.70 & 2.90 & 2.80 \\
\hline $\mathbf{T}_{7}$ & 7.52 & 8.50 & 8.01 & 5.50 & 6.00 & 5.75 & 2.90 & 3.80 & 3.35 \\
\hline $\mathbf{T}_{8}$ & 9.53 & 11.00 & 10.27 & 6.00 & 6.50 & 6.25 & 3.81 & 4.23 & 4.02 \\
\hline $\mathbf{T}_{9}$ & 7.10 & 8.00 & 7.55 & 5.00 & 5.30 & 5.15 & 2.90 & 2.90 & 2.90 \\
\hline$T_{10}$ & 9.30 & 10.80 & 10.05 & 5.81 & 6.30 & 6.06 & 3.71 & 4.10 & 3.90 \\
\hline$T_{11}$ & 2.60 & 2.70 & 2.65 & 2.30 & 2.80 & 2.55 & 1.80 & 1.90 & 1.85 \\
\hline$T_{12}$ & 4.00 & 4.80 & 4.40 & 3.10 & 3.70 & 3.40 & 2.80 & 2.90 & 2.85 \\
\hline F-test & $* *$ & $* *$ & $* *$ & $* *$ & $* *$ & $* *$ & $* *$ & $* *$ & $* *$ \\
\hline S.Em \pm & 0.20 & 0.22 & 0.15 & 0.27 & 0.24 & 0.18 & 0.20 & 0.15 & 0.13 \\
\hline C.D. @ $\overline{\mathbf{5} \%}$ & 0.58 & 0.66 & 0.43 & 0.80 & 0.71 & 0.52 & 0.59 & 0.44 & 0.36 \\
\hline
\end{tabular}

$\mathbf{T}_{\mathbf{1}}: 100 \% \mathrm{~K}$ through $\mathrm{KCl}+\mathrm{NP}$ through fertilizers for targeted yield of $20 \mathrm{tha}^{-1}$

$\mathbf{T}_{2}: 100 \% \mathrm{~K}$ through $\mathrm{K}_{2} \mathrm{SO}_{4}+\mathrm{NP}$ through fertilizers for targeted yield of $20 \mathrm{tha}^{-1}$

$\mathbf{T}_{3}: 100 \% \mathrm{~K}$ through Bio-K + balance NP through fertilizers for targeted yield of $20 \mathrm{tha}^{-1}$

$\mathbf{T}_{4}: 50 \% \mathrm{~K}$ through Bio-K $+50 \% \mathrm{~K}$ through $\mathrm{KCl}+$ balance $\mathrm{NP}$ through fertilizers for targeted yield of $20 \mathrm{tha}^{-1}$

$\mathbf{T}_{5}: 50 \% \mathrm{~K}$ through Bio-K $+50 \% \mathrm{~K}$ through $\mathrm{K}_{2} \mathrm{SO}_{4}+$ balance NP through fertilizers for targeted yield of $20 \mathrm{t} \mathrm{ha}^{-1}$

$\mathbf{T}_{6}: 100 \% \mathrm{~K}$ through $\mathrm{KCl}+\mathrm{NP}$ through fertilizers for targeted yield of $25 \mathrm{tha}^{-1}$
$\mathbf{T}_{7}: 100 \% \mathrm{~K}$ through $\mathrm{K}_{2} \mathrm{SO}_{4}+\mathrm{NP}$ through fertilizers for targeted yield of $25 \mathrm{t} \mathrm{ha}^{-1}$

$\mathbf{T}_{\mathbf{8}}: 100 \% \mathrm{~K}$ through Bio-K + balance NP through fertilizers for targeted yield of $25 \mathrm{t} \mathrm{ha}^{-1}$

$\mathbf{T}_{\mathbf{9}}: 50 \% \mathrm{~K}$ through Bio-K $+50 \% \mathrm{~K}$ through $\mathrm{KCl}+$ balance

targeted yield of $25 \mathrm{tha}^{-1}$

$\mathbf{T}_{10}: 50 \% \mathrm{~K}$ through Bio-K $+50 \% \mathrm{~K}$ through $\mathrm{K}_{2} \mathrm{SO}_{4}+$ balance NP through fertilizers for targeted yield of $25 \mathrm{tha}^{-}$

$\mathbf{T}_{11}:$ Recommended dose of fertilizers alone $(75: 75: 100 \mathrm{~kg}$ NPK ha ${ }^{-1}$ )

$\mathbf{T}_{12}$ :Package of Practice recommendation (UAS B) 
Table.2 Microbial population in soil after harvest of finger millet as influenced by site specific nutrient management in potato based sequential cropping system

\begin{tabular}{|c|c|c|c|c|c|c|c|c|c|}
\hline \multirow[t]{2}{*}{ Treatments } & \multicolumn{3}{|c|}{ Bacteria (x 10 Cfu g $\left.^{-1}\right)$} & \multicolumn{3}{|c|}{ Fungi $\left(x 1^{3} \mathrm{Cfu} \mathrm{g}^{-1}\right)$} & \multicolumn{3}{|c|}{ Actnomycetes $\left(\times 10^{2} \mathrm{Cfu} \mathrm{g}^{-1}\right)$} \\
\hline & 2017 & 2018 & Pooled & 2017 & 2018 & Pooled & 2017 & 2018 & Pooled \\
\hline $\mathbf{T}_{1}$ & 2.00 & 3.63 & 2.82 & 1.50 & 2.40 & 1.95 & 1.10 & 1.30 & 1.20 \\
\hline $\mathbf{T}_{2}$ & 3.80 & 4.00 & 3.90 & 2.00 & 2.50 & 2.25 & 1.20 & 1.40 & 1.30 \\
\hline $\mathbf{T}_{3}$ & 5.23 & 5.50 & 5.37 & 2.97 & 3.27 & 3.12 & 1.30 & 1.54 & 1.42 \\
\hline $\mathbf{T}_{4}$ & 5.20 & 5.23 & 5.22 & 1.60 & 2.37 & 1.99 & 1.22 & 1.40 & 1.31 \\
\hline $\mathbf{T}_{\mathbf{5}}$ & 5.00 & 6.40 & 5.70 & 4.10 & 4.70 & 4.40 & 1.20 & 1.80 & 1.50 \\
\hline $\mathbf{T}_{6}$ & 5.72 & 6.60 & 6.16 & 4.00 & 5.00 & 4.50 & 1.50 & 2.03 & 1.77 \\
\hline $\mathbf{T}_{7}$ & 8.00 & 9.00 & 8.50 & 5.20 & 5.90 & 5.55 & 2.00 & 2.40 & 2.20 \\
\hline $\mathbf{T}_{8}$ & 12.00 & 12.80 & 12.40 & 5.90 & 7.10 & 6.50 & 2.58 & 2.85 & 2.72 \\
\hline $\mathbf{T}_{9}$ & 7.62 & 8.50 & 8.06 & 4.40 & 5.40 & 4.90 & 1.80 & 2.01 & 1.91 \\
\hline $\mathbf{T}_{10}$ & 11.80 & 12.60 & 12.20 & 5.80 & 7.00 & 6.40 & 2.50 & 2.82 & 2.66 \\
\hline $\mathbf{T}_{11}$ & 2.10 & 2.90 & 2.50 & 1.50 & 2.30 & 1.90 & 1.00 & 1.20 & 1.10 \\
\hline $\mathbf{T}_{12}$ & 3.50 & 4.30 & 3.90 & 2.20 & 3.43 & 2.82 & 1.20 & 1.40 & 1.30 \\
\hline F-test & $* *$ & $* *$ & $* *$ & $* *$ & $* *$ & $* *$ & $* *$ & $* *$ & $* *$ \\
\hline S.Em \pm & 0.21 & 0.27 & 0.17 & 0.14 & 0.18 & 0.11 & 0.08 & 0.11 & 0.07 \\
\hline C.D. @ 5\% & 0.62 & 0.79 & 0.49 & 0.42 & 0.52 & 0.32 & 0.23 & 0.32 & 0.19 \\
\hline
\end{tabular}

Legend

$\mathrm{T}_{1}: 100 \% \mathrm{~K}$ through $\mathrm{KCl}+\mathrm{NP}$ through fertilizers for targeted yield of $20 \mathrm{t} \mathrm{ha}^{-1}$

$\mathrm{T}_{2}: 100 \% \mathrm{~K}$ through $\mathrm{K}_{2} \mathrm{SO}_{4}+\mathrm{NP}$ through fertilizers for targeted yield of $20 \mathrm{t} \mathrm{ha}^{-1}$

$\mathrm{T}_{3}: 100 \% \mathrm{~K}$ through Bio-K + balance NP through fertilizers for targeted yield of $20 \mathrm{t} \mathrm{ha}^{-1}$

$\mathrm{T}_{4}: 50 \% \mathrm{~K}$ through Bio- $\mathrm{K}+50 \% \mathrm{~K}$ through $\mathrm{KCl}$ + balance NP through fertilizers for targeted yield of $20 \mathrm{tha}^{-1}$

$\mathrm{T}_{5}: 50 \% \mathrm{~K}$ through Bio-K $+50 \% \mathrm{~K}$ through $\mathrm{K}_{2} \mathrm{SO}_{4}+$ balance NP through fertilizers for targeted yield of $20 \mathrm{tha}^{-1}$

$\mathrm{T}_{6}: 100 \% \mathrm{~K}$ through $\mathrm{KCl}+\mathrm{NP}$ through fertilizers for targeted yield of $25 \mathrm{tha}^{-1}$
$\mathrm{T}_{7}: 100 \% \mathrm{~K}$ through $\mathrm{K}_{2} \mathrm{SO}_{4}+\mathrm{NP}$ through fertilizers for targeted yield of $25 \mathrm{tha}^{-1}$

$\mathrm{T}_{8}: 100 \% \mathrm{~K}$ through Bio-K + balance NP through fertilizers for targeted yield of $25 \mathrm{tha}^{-}$

$\mathrm{T}_{9}: 50 \% \mathrm{~K}$ through Bio-K $+50 \% \mathrm{~K}$ through $\mathrm{KCl}+$ balance through fertilizers for targeted yield of $25 \mathrm{tha}^{-}$ $\mathrm{T}_{10}: 50 \% \mathrm{~K}$ through Bio-K $+50 \% \mathrm{~K}$ through $\mathrm{K}_{2} \mathrm{SO}_{4}+$ balance NP through fertilizers for targeted yield of $25 \mathrm{tha}^{-1}$

$\mathrm{T}_{11}$ :Recommended dose of fertilizers alone (75:75:100 kg NPK ha ${ }^{-1}$ )

$\mathrm{T}_{12}$ :Package of Practice recommendation (UAS B) 
Table.3 Microbial population in soil after harvest of field bean as influenced by site specific nutrient management in potato based sequential cropping system

\begin{tabular}{|c|c|c|c|c|c|c|c|c|c|}
\hline \multirow[t]{2}{*}{ Treatments } & \multicolumn{3}{|c|}{ Bacteria (x 10 $\left.{ }^{6} \mathrm{Cfu} \mathrm{g}^{-1}\right)$} & \multicolumn{3}{|c|}{ Fungi (x 10 ${ }^{4} \mathrm{Cfu} \mathrm{g}^{-1}$ ) } & \multicolumn{3}{|c|}{ 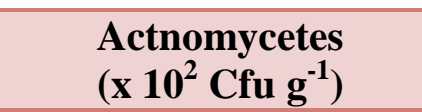 } \\
\hline & 2017 & 2018 & Pooled & 2017 & 2018 & Pooled & 2017 & 2018 & Pooled \\
\hline $\mathbf{T}_{1}$ & 6.00 & 7.63 & 6.82 & 4.00 & 4.87 & 4.43 & 3.00 & 3.30 & 3.15 \\
\hline $\mathbf{T}_{2}$ & 7.84 & 8.00 & 7.92 & 4.50 & 5.00 & 4.75 & 3.00 & 3.10 & 3.05 \\
\hline $\mathbf{T}_{\mathbf{3}}$ & 9.20 & 9.50 & 9.35 & 5.47 & 5.80 & 5.64 & 3.90 & 4.42 & 4.16 \\
\hline $\mathbf{T}_{4}$ & 6.22 & 7.23 & 6.73 & 4.10 & 4.87 & 4.49 & 2.52 & 2.91 & 2.72 \\
\hline $\mathbf{T}_{\mathbf{5}}$ & 9.00 & 10.40 & 9.70 & 6.60 & 7.20 & 6.90 & 3.80 & 3.90 & 3.85 \\
\hline $\mathbf{T}_{6}$ & 9.72 & 10.60 & 10.16 & 6.50 & 7.50 & 7.00 & 3.72 & 4.10 & 3.91 \\
\hline $\mathbf{T}_{7}$ & 12.00 & 13.00 & 12.50 & 7.70 & 8.40 & 8.05 & 4.50 & 4.80 & 4.65 \\
\hline $\mathbf{T}_{8}$ & 16.00 & 16.72 & 16.36 & 8.20 & 10.00 & 9.10 & 5.40 & 5.95 & 5.68 \\
\hline $\mathbf{T}_{9}$ & 11.73 & 12.50 & 12.12 & 6.90 & 7.90 & 7.40 & 3.81 & 4.23 & 4.02 \\
\hline$T_{10}$ & 15.50 & 16.43 & 15.97 & 8.05 & 9.90 & 8.98 & 5.32 & 5.85 & 5.58 \\
\hline $\mathbf{T}_{11}$ & 6.10 & 6.90 & 6.50 & 4.00 & 4.80 & 4.40 & 2.70 & 3.00 & 2.85 \\
\hline $\mathbf{T}_{12}$ & 7.50 & 8.30 & 7.90 & 4.72 & 5.93 & 5.32 & 2.90 & 3.30 & 3.10 \\
\hline F-test & $* *$ & $* *$ & $* *$ & $* *$ & $* *$ & $* *$ & $* *$ & $* *$ & $* *$ \\
\hline S.Em \pm & 0.27 & 0.12 & 0.15 & 0.19 & 0.26 & 0.16 & 0.17 & 0.22 & 0.14 \\
\hline C.D. @ 5\% & 0.80 & 0.35 & 0.42 & 0.28 & 0.77 & 0.47 & 0.48 & 0.64 & 0.39 \\
\hline
\end{tabular}

Legend:

$\mathrm{T}_{1}: 100 \% \mathrm{~K}$ through $\mathrm{KCl}+\mathrm{NP}$ through fertilizers for targeted yield of $20 \mathrm{tha}^{-1}$

$\mathrm{T}_{2}: 100 \% \mathrm{~K}$ through $\mathrm{K}_{2} \mathrm{SO}_{4}+\mathrm{NP}$ through fertilizers for targeted yield of $20 \mathrm{tha}^{-1}$

$\mathrm{T}_{3}: 100 \% \mathrm{~K}$ through Bio-K + balance NP through fertilizers for targeted yield of $20 \mathrm{tha}^{-1}$

$\mathrm{T}_{4}: 50 \% \mathrm{~K}$ through Bio- $\mathrm{K}+50 \% \mathrm{~K}$ through $\mathrm{KCl}$ + balance NP through fertilizers for targeted yield of $20 \mathrm{t} \mathrm{ha}^{-1}$

$\mathrm{T}_{5}: 50 \% \mathrm{~K}$ through Bio-K $+50 \% \mathrm{~K}$ through $\mathrm{K}_{2} \mathrm{SO}_{4}+$ balance NP through fertilizers for targeted yield of $20 \mathrm{tha}^{-1}$

$\mathrm{T}_{6}: 100 \% \mathrm{~K}$ through $\mathrm{KCl}+\mathrm{NP}$ through fertilizers for targeted yield of $25 \mathrm{tha}^{-1}$

Fungal population was significantly higher with $100 \% \mathrm{~K}$ through Bio-K + balance NP through fertilizers for targeted yield of $25 \mathrm{t}$ $\mathrm{ha}^{-1}\left(6.50 \times 10^{3} \mathrm{Cfu} \mathrm{g}^{-1}\right)$ and it was found to be on par with $50 \% \mathrm{~K}$ through Bio-K $+50 \% \mathrm{~K}$ through $\mathrm{K}_{2} \mathrm{SO}_{4}+$ balance $\mathrm{NP}$ through fertilizers for targeted yield of $25 \mathrm{t} \mathrm{ha}^{-1}(6.40 \mathrm{x}$ $\left.10^{3} \mathrm{Cfu} \mathrm{g}^{-1}\right)$ as compared to other treatments.
$\mathrm{T}_{7}: 100 \% \mathrm{~K}$ through $\mathrm{K}_{2} \mathrm{SO}_{4}+\mathrm{NP}$ through fertilizers for targeted yield of $25 \mathrm{tha}^{-1}$

$\mathrm{T}_{8}: 100 \% \mathrm{~K}$ through Bio-K + balance NP through fertilizers for targeted yield of $25 \mathrm{t} \mathrm{ha}^{-}$

$\mathrm{T}_{9}: 50 \% \mathrm{~K}$ through Bio-K $+50 \% \mathrm{~K}$ through $\mathrm{KCl}+$ balance

through fertilizers for targeted yield of $25 \mathrm{tha}^{-}$

$\mathrm{T}_{10}: 50 \% \mathrm{~K}$ through Bio-K $+50 \% \mathrm{~K}$ through

$\mathrm{K}_{2} \mathrm{SO}_{4}+$ balance NP through fertilizers for targeted yield of $25 \mathrm{t} \mathrm{ha}^{-1}$

$\mathrm{T}_{11}$ :Recommended dose of fertilizers alone (75:75:100 kg NPK ha ${ }^{-1}$ )

$\mathrm{T}_{12}$ :Package of Practice recommendation (UAS B)

Significantly lower fungal population was observed in recommended dose of fertilizers alone $\left(1.90 \mathrm{x} \quad 10^{3} \mathrm{Cfu} \mathrm{g}^{-1}\right) \quad$ (Table 2). Significantly higher actinomycetes population was observed in soil after harvest of finger millet in plot which received $100 \% \mathrm{~K}$ through Bio-K + balance NP through fertilizers for targeted yield of $25 \mathrm{tha}^{-1}(2.72 \mathrm{x}$ 
$10^{2} \mathrm{Cfu} \mathrm{g}^{-1}$ ) and it was on par with $50 \% \mathrm{~K}$ through Bio-K $+50 \% \mathrm{~K}$ through $\mathrm{K}_{2} \mathrm{SO}_{4}+$ balance NP through fertilizers for targeted yield of $25 \mathrm{t} \mathrm{ha}^{-1}\left(2.66 \times 10^{2} \mathrm{Cfu} \mathrm{g}^{-1}\right)$ as compared to other treatments. Significantly lower actinomycetes population was observed in recommended dose of fertilizers alone $\left(1.10 \times 10^{2} \mathrm{Cfu}^{-1}\right)$. The higher microbial population might have been possible owing to enhanced supply of available food and energy to these organisms in soil through supply BioK. Microbes are known to increase the availability of immobile micronutrients such as $\mathrm{Fe}, \mathrm{Zn}, \mathrm{Cu}$ and $\mathrm{Mn}$ along with major nutrient phosphorus through mobilization process. The Improved microbial properties due to improve the metabolic activities of plant, increased in organic matter content of the soil resulting in a significant increase microbial activity. The similar results are in line with the findings of Anand (2010). This could result in better growth of microorganism which in turn resulted in more release of root exudates which acts as a source of carbon and energy for microorganisms.

Significantly higher bacterial population was observed in soil after harvest of field bean in plot which received $100 \% \mathrm{~K}$ through Bio-K + balance NP through fertilizers for targeted yield of $25 \mathrm{t} \mathrm{ha}^{-1}\left(16.36 \times 10^{6} \mathrm{Cfu} \mathrm{g}^{-1}\right)$ and it was on par with $50 \% \mathrm{~K}$ through Bio-K +50 $\% \mathrm{~K}$ through $\mathrm{K}_{2} \mathrm{SO}_{4}+$ balance NP through fertilizers for targeted yield of $25 \mathrm{t} \mathrm{ha}^{-1}$ (15.97 $\mathrm{x} 10^{6} \mathrm{Cfu} \mathrm{g}^{-1}$ ) as compared to other treatments.. Significantly lower bacterial population was observed in recommended dose of fertilizers alone $\left(6.50 \times 10^{6} \mathrm{Cfu} \mathrm{g}^{-1}\right)$ (Table 3). Fungal population was significantly higher with $100 \% \mathrm{~K}$ through Bio-K + balance NP through fertilizers for targeted yield of $25 \mathrm{t} \mathrm{ha}^{-1}\left(9.10 \times 10^{4} \mathrm{Cfu} \mathrm{g}^{-1}\right)$ and it was found to be on par with $50 \% \mathrm{~K}$ through Bio-K $+50 \% \mathrm{~K}$ through $\mathrm{K}_{2} \mathrm{SO}_{4}+$ balance NP through fertilizers for targeted yield of $25 \mathrm{t}$ $\mathrm{ha}^{-1}\left(8.98 \times 10^{4} \mathrm{Cfu} \mathrm{g}^{-1}\right){ }^{\text {as }}$ compared to other treatments. Significantly lower fungal population was observed in recommended dose of fertilizers alone $\left(5.32 \times 10^{4} \mathrm{Cfu} \mathrm{g}^{-1}\right)$ (Table 3).

Significantly higher actinomycetes population was observed in soil after harvest of field bean in plot which received $100 \% \mathrm{~K}$ through Bio-K + balance NP through fertilizers for targeted yield of $25 \mathrm{t} \mathrm{ha}^{-1}\left(5.68 \times 10^{2} \mathrm{Cfu} \mathrm{g}^{-1}\right)$ and it was on par with $50 \% \mathrm{~K}$ through Bio-K $+50 \% \mathrm{~K}$ through $\mathrm{K}_{2} \mathrm{SO}_{4}+$ balance $\mathrm{NP}$ through fertilizers for targeted yield of $25 \mathrm{t}$ $\mathrm{ha}^{-1}\left(5.58 \times 10^{2} \mathrm{Cfu} \mathrm{g}^{-1}\right)$ as compared to other treatments. Significantly lower actinomycetes population was observed in recommended dose of fertilizers alone $\left(2.85 \times 10^{2} \mathrm{Cfu} \mathrm{g}^{-1}\right)$ (Table 3). The microbial analysis pertaining to bacteria, fungi and actinomycetes population in soil after harvest of field bean differed significantly due to supply of nutrients through site specific nutrient management and Bio-K which improved the organic matter content and nutrient status of soil in potato based sequential cropping system resulted in higher microbial activity (Anand 2010 and Lingaraju, 2018).

In conclusion potato-finger millet and potatofield bean cropping systems, supply of potassium through combination of Bio-K and SOP under SSNM approach are beneficial to increase the microbial population in the above cropping systems.

\section{References}

ANAND, S. R., 2010, Site specific nutrient management (SSNM) for maximization of crop productivity in southern Karnataka. Ph.D. Thesis, University of Agricultural Sciences, Bangalore.

ANONYMOUS, 2018a, Annual. report, Nutritional security, livelihoods and climate resilience in Asia. Lima (Peru). 
CIP. $4 p$

BILSKI, J. J., NELSON AND CONLON, R. L., 1988. The response of four potato cultivars to chloride salinity, sulfate salinity and calcium in pot experiments. American Potato J., 65: 85-90.

CORNE KEMPENAAR, THOMAS BEE, JOHAN BOOIJ, FRITS VAN EVERT, JEAN-MARIE MICHIELSEN AND CORNE KOCKS, 2017, Advances in variable rate technology application in potato in the Netherlands. Potato Res., 60: 295-305.

CUI Z, L., ZHANG H, Y., CHEN, X. P., ZHANG, C, C., MA, W. Q. AND HUANG C. D., 2018. Pursuing sustainable productivity with millions of smallholder farmers. Nature (London), 555: 363- 366.

DREWNOWSKI, A, 2013. New metrics of affordable nutrition: Which vegetables provide the most nutrients for least cost. J. Academy Nutrition Dietetics, 113: 1182-1187.

FELIPE DE MENDIBUR, 2020, Agricolae: Statistical procedures for agricultural research. $\mathrm{R}$ package version 1.3-3.

GOMEZ, K. A. AND GOMEZ, A. A., 1984, Statistical procedures for Agricultural Research, 2 ${ }^{\text {nd }}$ Edition A Wiley Inter
Science Publication. New York (USA).

LINGARAJU, N. N., 2018, response of maize-cowpea sequence to treated sugarmill effluent irrigation under different nutrient and moisture regimes in Cauvery command area. Ph.D. Thesis, University of Agricultural Sciences, Bangalore.

MCGILL, C. R., A. C. KURILICH, AND DAVIGNON, J., 2013. The role of potatoes and potato components in cardiometabolic health: A review. Annals of Medicine, 45: 467-473.

SOMANI, A. K. AND SHEKHAWAT, G. S., 1988, Influence of levels and sources of nitrogen on the development of bacterial soft-rot in potato tubers. Phytopathology, 41: 238-240.

WESTERMANN, D. T., TINDALL, T. A., JAMES, D.W. AND HURST, R. L., 1994, Nitrogen and potassium fertilization of potatoes: yield and specific gravity. American Potato J., 71: 417-431.

YLI-HALLA, M., E. VIIKARI AND PALONEN, J., 1987, Quantity and quality of potato yield as influenced by unbalanced and excessive fertilization. Finland J. Agric. Sci., 59: 131-139.

\section{How to cite this article:}

Manjunatha, M. H., S. Bhaskar, B. C. Mallesha, N. N. Lingaraju and Basavaraja, B. 2021. Effect of Site Specific Nutrient Management in Potato based Sequential Cropping Systems on Microbial Population Dynamics. Int.J.Curr.Microbiol.App.Sci. 10(02): 1361-1368. doi: https://doi.org/10.20546/ijcmas.2021.1002.162 